\title{
$\eta$ Carinae: The optical flare-like events during low-excitation passages
}

\author{
A. M. van Genderen ${ }^{1}$, C. Sterken ${ }^{2, \star}$, and W. H. Allen ${ }^{3}$ \\ 1 Leiden Observatory, Postbus 9513, 2300RA Leiden, The Netherlands \\ 2 Astronomy Group, Vrije Universiteit Brussel, Pleinlaan 2, 1050 Brussels, Belgium \\ 3 Alvine Estate, Vintage Lane, RD 3, Blenheim, New Zealand
}

Received 4 November 2002 / Accepted 23 April 2003

\begin{abstract}
The 1992.5 and 1998.0 low-excitation events of the possible binary in the core of $\eta$ Carinae show a conspicuous flare-like event in optical and near-ultraviolet light. These events last a couple of months and have a light amplitude of $0 .{ }^{\mathrm{m}} 1-0.2$ in $V$. Considering the presence of a dense (bi-polar) stellar wind, one explanation could be that some type of a hot area is created by an increased mass flow from the S Dor primary near the hypothetical periastron. These flare-like events are terminated by sharp dips. The magnitude differences between the underlying light source and the central light ("Component A") are derived. It turns out that they are fainter than Component $\mathrm{A}$ by $\sim 2 \mathrm{~m}$. The luminosity could be of the order of $10^{5} L_{\odot}$. It was possible to derive two new continuum values in the near-UV, reinforcing the impression derived from the HST fluxes that Component A has a very strong radiation peak in the Balmer continuum. This can probably be explained by the strong emission of the stellar wind.
\end{abstract}

Key words. technique: photometric - stars: individual: $\eta$ Carinae - stars: variables: general - stars: supergiants

\section{Introduction}

The central object of $\eta$ Car might contain a massive collidingwind binary: an S Dor variable (or LBV) which was responsible for the $\mathrm{S}$ Dor (SD)-type eruption creating the bipolar nebula (or Homunculus), and a hot, but much fainter companion with a period of $5.5 \mathrm{yr}$ (Damineli 1996). A large body of literature suggests that $\eta$ Car could contain an interacting collidingwind binary and some studies discuss the possible presence of an equatorial (circumbinary) disk (e.g. Damineli et al. 1998; Davidson 1999; van Genderen et al. 1999; Duncan et al. 1999; Whitelock \& Laney 1999; Stevens \& Pittard 1999; Corcoran et al. 2000, 2001a,b; Feast et al. 2001; Hillier et al. 2001; Pittard \& Corcoran 2002; Duncan \& White 2003). Nevertheless, problems with a binary model were outlined as well by Stevens \& Pittard (1999), Davidson et al. (2000) and Viotti et al. (2002). One peculiarity is that the brightness of the central object increased by a factor of two in 1999 compared to 1998 (Davidson et al. 1999), while the entire Homunculus brightened less, see the light curve by Sterken et al. (2001b). On account of a possible $200 \mathrm{~d}$ oscillation superimposed on a $5.5 \mathrm{yr}$ modulation in the Balmer continuum, the existence of a second companion has been tentatively proposed (van Genderen et al. 1999; 2001, in the latter paper the peculiar phenomena in the UV gave rise to the designation "The near-UV enigma").

Send offprint requests to: A. M. van Genderen, e-mail: genderen@strw.leidenuniv.nl

^ Research Director, Belgian Fund for Scientific Research (FWO).
The SD-type instability (the SD phases ${ }^{1}$, i.e. the non-eruptive light variations) of the primary is to some extent influenced by a possible tidal modulation resulting in maxima near the "low-excitation events", the hypothetical periastron passages (PPs) (van Genderen et al. 2001, see also the NIR curves of Whitelock \& Laney 1999). This does not exclude other, usually less pronounced, maxima between the PPs since the SD phases also show shorter cycles than $5.5 \mathrm{yr}$.

The variable emission lines will certainly play a part in the optical variability. It appears that during the "low" states (around PP) emission lines (in particular the many highexcitation lines) are weaker than during the "high" states (outside PP) (Zanella et al. 1984; Damineli et al. 1998; Wolf et al. 1999). Thus, the light maxima during the PPs are likely due to the SD-activity of the S Dor primary (van Genderen et al. 2001).

During the PPs of 1987.0 and 1998.0 light enhancements and dips were found with $0 .{ }^{\mathrm{m}} 1-0.2$ amplitudes in the optical (van Genderen et al. 1999, 2001; Sterken et al. 1999b) and in

\footnotetext{
1 It should be noted that in our previous papers, as well as in papers by other authors, the SD-activity of $\eta$ Car was described as a kind of "eruption" with an opaque-shell ejection. In fact it is probably a cyclic pulsation phenomenon in the first place, while the massloss rate does vary, though generally not dramatically. Therefore, we speak of "SD phases". Massive shell ejections only occur a few times in the lifetime of an S Dor star and are designated "SD eruptions" (van Genderen 2001). In the case of $\eta$ Car the 1843 eruption is sometimes called the Great Eruption (Davidson \& Humphreys 1997).
} 
the NIR (Whitelock \& Laney 1999). The optical series near the 1992.5 PP showed a long gap without data. We have found that one of us (WHA) still had a number of unpublished $U B V$ observations made from 1989 until 1993. A part of this set, concentrated around the PP, will be discussed in the present paper.

\section{The value of integrated photometry}

In discussing photometry of the entire Homunculus reflection nebula, with the purpose to extract some information on the central star(s), the following points should be kept in mind. The central stellar light reaching us directly is weakened by the central dust shells and by the wall of the SE lobe. As a result, it is much weaker than at the lobes of the Homunculus which most likely have a much clearer line-of-sight to the central light source. However, the SE lobe, the brightest of the two, adds a great deal of scattered light to the central stellar light, and since this lobe is oriented towards us, light-time effects are negligible. For further background see van Genderen et al. (1999).

Yet, part of the integrated light should consist of smearedout and multiple reflections inside the lobes with time delays of weeks to months; however, in view of the detailed light variations (phase-locked and quasi-periodic) and of the fact that the variations of the $\mathrm{H} \beta$ index are precisely in anti-phase with the $\alpha$ Cyg-type variations, that contribution is certainly not dominant (Sterken et al. 1996; van Genderen et al. 1995, 1999, 2001), but might cause a reduction of the light amplitudes. Considering the amplitudes of the $\alpha$ Cyg-type oscillations, a rough estimate for the amount of reduction is a factor of two.

\section{The UBV observations}

The observations were obtained with a private $32-\mathrm{cm} \mathrm{f15}$ Dall Kirkham telescope situated in Raparaura, Marlborough, New Zealand. The telescope is mounted in a 5-m dome and was equipped with a professionally made photoelectric photometer and Johnson $U B V$ filter system. The photometric system was periodically calibrated using the standard E region stars (Menzies et al. 1989).

The observations of $\eta$ Car were made with respect to the comparison star q Car $=\operatorname{HD} 89388(V=3.36, B-V=1.54$, $U-B=1.67)$. It turned out from our photometry that q Car is slightly variable by at most $0 \mathrm{~m} .2$ and that it was a known variable star and designated as V337 Car in the 4th edition of the GCVS, with a magnitude range of 3.36-3.44, Sp = K3II (Kholopov et al. 1985), or K3IIa (Hoffleit 1982). The check star was p Car $=\operatorname{HD} 91465(V=3.31, B-V=-0.10$, $U-B=-0.71$ ) and is a Be star (Slettebak 1982), Sp = B4Vne (Hoffleit 1982). Cousins \& Warren (1963) noted a small, irregular light variation and in the GCVS the star is called PP Car with $V=3.27-3.37$. Fortunately, a third star was frequently observed together with $\eta$ Car: HD $93070(V=4.562, B-V=$ 1.699, $U-B=1.856$, HD Sp $=$ K5, or K4III by Hoffleit 1982) which was constant within 0. . 1 . (It must be noted that the latter is listed as NSV 4951 in the New Catalogue of Suspected Variables, Kukarkin et al. 1982). However, we doubt whether that is a correct identification. Although the coordinates agree, the magnitude does not: 3.17-3.34, while our $V$ magnitude confirms the one given above $(V=4.562)$, which is also consistent with the photometric magnitude from the HD catalogue: 4.5. Therefore, the effect of the variations of $q$ and $p$ Car on the light variation of $\eta$ Car could largely be eliminated. Although these data are not as precise as we would wish, they illustrate that a flare-like event similar to the one observed in 1998.0 was present. It should be noted that the $U B V$ photometry cited above is from Mermilliod et al. (1997).

The photometric reductions were made with an on line Apple IIe automated system and each $U, B$ and $V$ measure is the average of three 10 -second integrations. Each $V, B-V$ and $U-B$ data point is the average of three sets of $U B V$ observations relative to the comparison star. A check star was also measured. The Julian date was calculated from the mid point time of each set of observations (Allen 1989).

The estimated internal errors amount to \pm 0.04 in $V, \pm 0.02$ in $B-V$ and \pm 0 . 04 in $U-B$, but due to the small variations of the comparison and check star the total errors might be twice as large. Due to the emission spectrum of $\eta$ Car and the red comparison stars, some $U-B$ values might show systematic errors up to 0.2 depending on atmospheric conditions and airmass.

\section{The light and colour curves}

Figure 1 shows the light and colour curves in the NIR and in the optical of $\eta$ Car in various photometric systems between 1990.5 and 1993.5. The four panels at the top show the JHKL light curves of Whitelock et al. (1994). The errors correspond to 0.03 . The three other panels depict the $U B V$ photometry as crosses. The estimated errors amount to 0 . 04 in $V$, 0 m02 in $B-V$ and 0.05 in $U-B$. The observations in the Walraven VBLUW system (van Genderen et al. 1994a) and Strömgren $u v b y$ system (Sterken et al. 1995; Manfroid et al. 1994; van Genderen et al. 1995) are represented by dots. These dots have been shifted in vertical direction to match our more or less equivalent Johnson $U B V$ photometric parameters (thus, $B-V$ versus Walraven $V-B$ and Strömgren $b-y$, and $U-B$ versus Walraven $B-U$ and Strömgren $u-v$ ). The time series between HJD 2448900 and HJD 2449200 (uvby system) are so dense, that they are shown as a limited number of dots.

The square represents a small group of UBVRI observations made at the SAAO by D. Kilkenny in 1991 (van Genderen et al. 1995). It appears that there is a good match in $V$, but our $B-V$ and $U-B$ color indices turn out to be too blue by 0.05 and 0.25 , respectively. The SAAO $U-B=0.45$ is consistent with $U-B$ values obtained by Feinstein \& Marraco (1974) taking a slight blueing of $\eta$ Car into account and considering the very slow trend in similar colour indices of the $V B L U W$ system between the early seventies and the early nineties of the 20 th century. Therefore, we have translated our $U-B$ observations, as well as the data in the more or less equivalent colour indices of other systems to the SAAO data. The same has been done for the $B-V$ colour index. The two arrows in the $V$ diagram indicate the PPs according to the ephemeris of Corcoran et al. (2001a,b), at the left, and of Damineli et al. (2000), at the right. Figure 2 shows the $y$ and $b$ light curves of the 1998.0 event as obtained by Sterken et al. (1999b). The arrows have the same meaning as those in Fig. 1. Geneva magnitudes confirm the flare-like event but are not shown because 


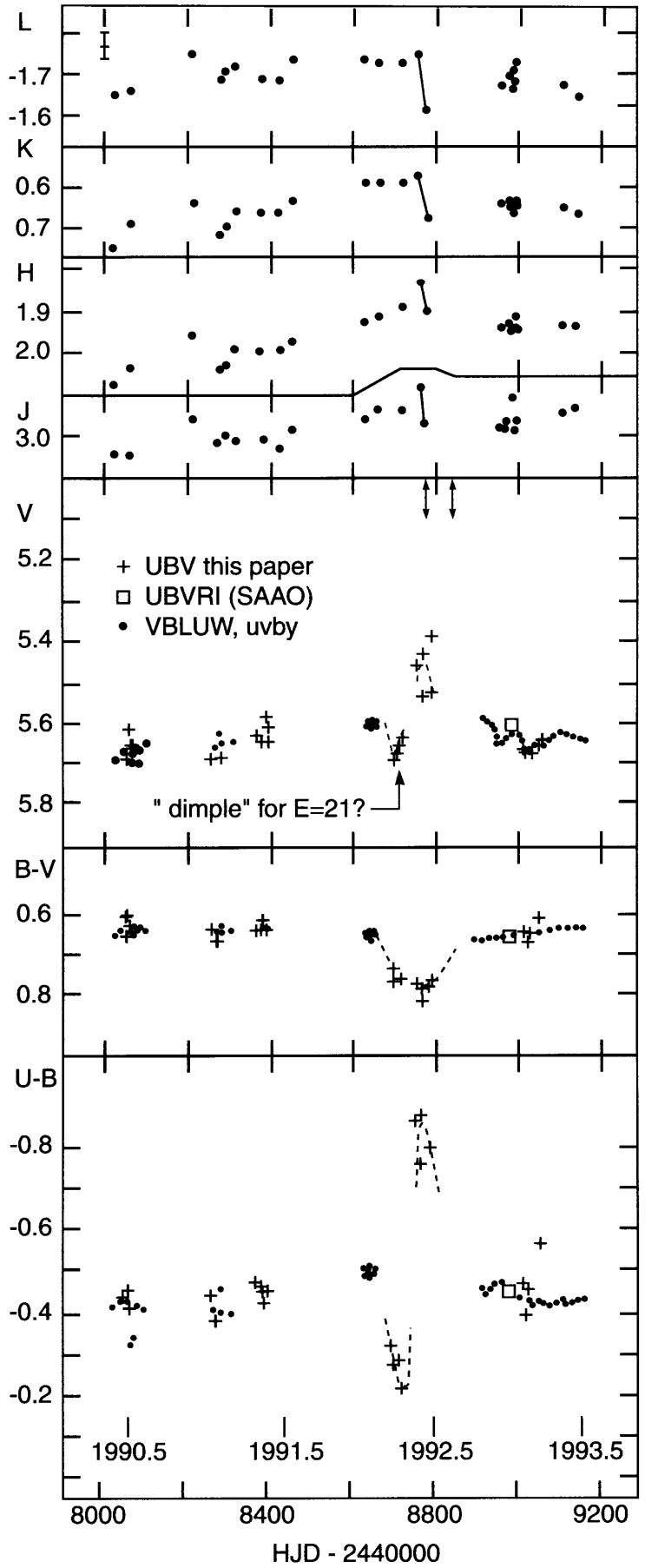

Fig. 1. The light and colour curves of $\eta$ Car around the 1992.5 periastron passage (PP) obtained in various photometric systems. The four panels at the top: JHKL light curves (Whitelock et al. 1994), error bar: two times the upper limit for the errors in $J H K$, for $L$ somewhat larger. The three lower panels: $V B L U W$ and $u v b y$ data points (dots) matched onto the $U B V$ data points (crosses). The two arrows in the fifth panel indicate the date of the computed PP according to two different authors (see text).

they were obtained in a $V$ band much wider than $y$ (the particular problems concerning photometry of $\eta$ Car with different photometric systems have been discussed by Sterken et al. 1999a,b, 2001a,b; Sterken 2000).

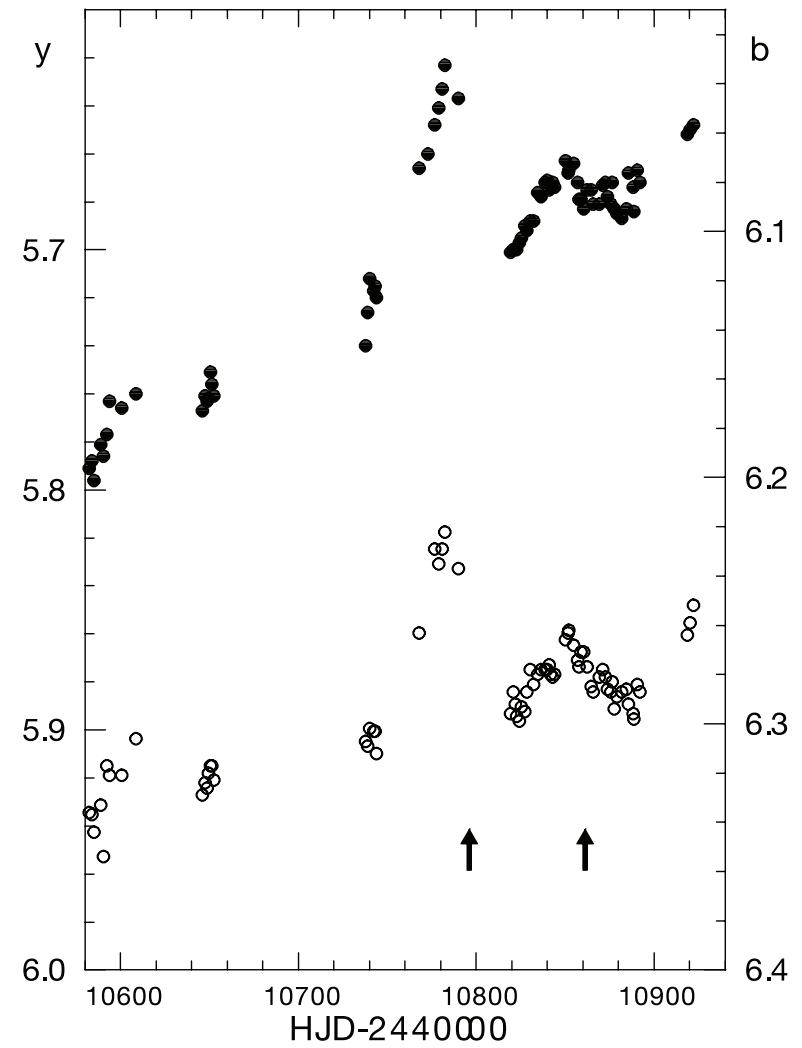

Fig. 2. $y$ and $b$ light curve based on the data of Sterken et al. (1999b). The arrows indicate the date of the computed PP.

\section{The analysis}

The brightness between HJD 2448000 and HJD 2448400 shows the same slowly-rising trend in all photometric bands, with a maximum around 1992.5 (ignoring the peak, see Fig. 1). These maxima are more or less synchronized with the PPs (Sect. 1).

The beginning of a dip between HJD 2448705 and HJD 2448728 is obvious in the $U B V$ data. Declines in $V, B-V$ and $U-B$ amount to respectively $0.07,0 \mathrm{~m} .15$ and 0 . 3 . The last of the four observations is only 3 days from such a predicted minimum or "dimple" computed with the ephemeris given by van Genderen et al. (1994a) for $E=21$ (in the latter paper these features were designated as type IV variations). The origin of the dimples is quite obscure. They are generally deepest in the UV, with some exceptions. Their precise duration could never be established because of gaps in the time series and their rare appearances, but our impression is that they last about a week. We emphasize that the possible periodicity of this phenomenon $\left(52^{\mathrm{d}} 38\right)$ is of a quite different nature than that of the $\alpha$ Cyg-type variations of the $\mathrm{S}$ Dor star $\left(58^{\mathrm{d}} 56\right.$, van Genderen et al. 1995; Sterken et al. 1996). It is not excluded that $52^{\mathrm{d}} 38$ is not the correct cycle length, it might be that this period even is twice as long. One NIR data point (HJD 2448721) coincides with this feature, but it does not show any sign of a dip. This may be due to the wavelength dependency where the dips are often nearly absent at longer wavelenghts.

Over a month later, during the PP, between HJD 2448763 (20 May 1992) and HJD 2448797 (23 June 1992), the visual 
brightness had increased by $\sim 0$. 15 (relative to the pre-dimple level). $B-V$ became redder by nearly the same amount, indicating that the rise in $B$ was smaller, and $U-B$ became bluer by 0.35 . Thus, this flare-like event shows a peculiar trend of the amplitudes from $V$ to $U: 0.15,0.05$ and 0.40 , respectively. Then, a few months later, the brightness is back to normal and slowly declining. Figure 2 shows that the 1998.0 flarelike event is superimposed on a rising branch. The amplitude in $y$ amounts to $0 . \mathrm{m} 1$, in $b$ it is slightly less. The feature lasts about two months.

Only two data points in the NIR (Fig. 1) appear to coincide with the optical flare (represented by five data points between HJD 2448763 and HJD 2448 797, or with the ephemeris of Damineli et al. (2000), between phase 0.96 and 0.98): at HJD 2448761 and HJD 2448783 (connected by a line). Our last data point was obtained $44^{\mathrm{d}}$ before the PP. Thus, while the optical flare is still present, the first NIR magnitude only suggests a very weak event, and the second shows a steep decline ( 0.1 in $J H K$ and 0.14 in $L$ ). This might cast doubt on one of the two data sets, were it not that the feature of 1987.0 demonstrates that time differences for such features between the NIR and the optical light do occur.

Figure 3 shows this feature during and after the 1987.0 PP in detail, with the nine light curves (JHKL and VBLUW) in order of decreasing wavelength. The tick marks on the left are steps of 0.02 . Considering the mutual consistency of the $J H K L$ light curves, the error bar is certainly an upper limit. The two error bars adjacent to the $V B L U W$ curves represent the range of the mean errors from $V$ to $W$, respectively. The meaning of the arrows is the same as for Figs. 1 and 2. The start of the 1987.0 feature in the NIR suggests an egress phase after an eclipse or obscuration (as advocated by Whitelock \& Laney 1999 and Feast et al. 2001) followed by the abnormally sharply-peaked event, unusual for S Dor variables. The precise start of the egress has probably been missed by both campaigns, but it is obvious that the maximum in the NIR was reached about three months before it occurred in the optical light. When the optical reached maximum, the NIR had declined to a lower level. A significant time delay was also present between the X-ray dip and the optical and NIR dips during the 1998.0 PP possibly due to geometrical effects (van Genderen et al. 1999, 2001).

\section{Discussion}

It appears that the flare-like events during the PPs of 1992.5 and 1998.0 were no accidental light fluctuations as already suggested before (van Genderen et al. 1999, 2001). The 1987.5 event could also be the possible remains of a feature with the same physical origin. The phenomenon is presumably not a flash of stellar light. The following scenario is plausible: while the hypothetical companion is approaching, the S Dor star expands by the influence of tidal interaction on its cyclic instability (see the visual maxima in Fig. 3 by van Genderen et al. 2001; for those in the NIR see Whitelock et al. 1994 and Whitelock \& Laney 1999), and the star loses more mass. Indeed, an increasing mass-loss rate around the PP by a factor

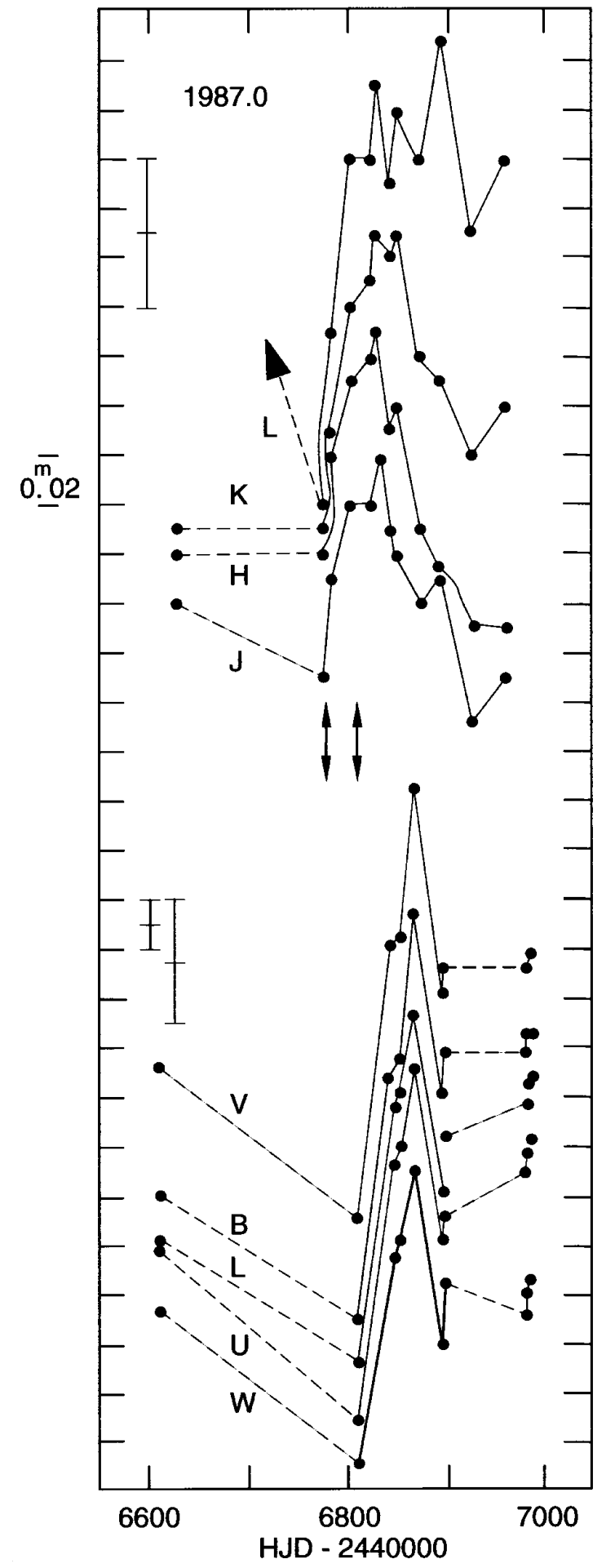

Fig. 3. The feature following the PP of 1987.0 with nine light curves in order of decreasing wavelength from top to bottom. The tick marks on the left are steps of 0.02 . Error bars at the top are the same as in Fig. 1. The two error bars below correspond to $2 \times$ the mean error for $V$ (smallest bar) to $W$ (largest bar).

of 20 to $\leq 6 \times 10^{-3} M_{\odot} \mathrm{y}^{-1}$, is necessary to improve the model fit around X-ray minimum (Corcoran et al. 2001a,b).

This mass flow will collide with other gas complexes, resulting in a heating and ionization process. Duncan \& White (2003) have found evidence that during the 1998.0 PP, material 
was tidally lifted from the primary star into its equatorial disk. Whether this has anything to do with the flare is not clear.

That influential gaseous structures undergo considerable physical changes near the PPs, has been proven e.g. by the sudden steep declines of the Balmer continuum radiation by 10 $20 \%$ after the PPs. This behaviour seems to modulate with the $5.5 \mathrm{yr}$ period (van Genderen et al. 1999, 2001).

We apply the following relation, and assuming that all kinetic energy is transformed into radiation at or close to the stellar surface of a possible companion (Lang 1998): $L_{\text {accr }}=$ $10^{34} \dot{M}\left(M / M_{\odot}\right)\left(R_{\odot} / R\right)$ W. We assume a main-sequence companion with $30 M_{\odot}, 7 R_{\odot}$ and a concentrated mass stream during the PP (e.g. through $L_{1}$ ) with $\dot{M}=10^{-3} M_{\odot} \mathrm{yr}^{-1}$, $L_{\text {accr }}=10^{5} L_{\odot}$.

A quantitative discussion of the true energy of the source of the flare-like event (hereafter called "flare") is difficult, in particular because the internal extinction inside the Homunculus, as well as the extinction law, is hard to determine. The interstellar foreground extinction is much less of a problem. However, it is possible to derive at least the magnitude differences between the central light source in a very small field with a diameter of a few arcsec, hereafter called "Component A" (Davidson et al. 1995) and the source of the flare. This can be done by subtracting the maximum flux of the flare from the underlying flux. It appears that the flare of 1992.5 is fainter than the Homunculus by 1,3 and 2 mag in the near-UV, the blue and the visual, respectively. For 1998.0 these quantities amount to $\sim 2.5$ for the blue and the visual, not much different from those of the 1987.5 feature including the near-UV.

Thus, we find a brightness difference of a factor of 10 . We assume that this difference also applies between Component $\mathrm{A}$ (the light source of the Homunculus) and the flare if directly seen by the observer (instead of via the Homunculus). Based on the limited information on the spectrum of the flare, it is hardly possible to derive a reliable luminosity, but that it should be at least of the order of $10^{5} L_{\odot}$ seems plausible. It is also not possible to assign a reliable $M_{\mathrm{v}}$ to the flare, because $M_{\mathrm{v}}$ for the S Dor primary ranges between -7 and -9 (van Genderen 2001), while applying a visual reddening $A_{\mathrm{v}}=6.1$, Davidson et al. (1995) find $M_{\mathrm{v}}=-9.5$ with an uncertainty of probably $1 \mathrm{~m} .5$.

We determined the magnitude differences between the Homunculus and Component A using HST fluxes at nine wavelengths (the tenth at $1460 \AA$ is very unreliable, Davidson et al. 1995), assuming that in the small field of a few 0.1 back - and foreground light can be neglected. The wavelength range is from $5445 \AA$ to $3535 \AA$, with a gap between $3645 \AA$ and $3535 \AA$. Ground-based $U B V$ and $V B L U W$ photometry of the Homunculus is available at dates not too distant in time from the HST fluxes of Component A (June 1991, $U B V$ and March 1991, VBLUW). The HST fluxes were obtained in August 1991. The light variation between these two dates is negligible (Fig. 1).

Magnitudes $U B V$ and $V B U$ (from the $V B L U W$ system) were derived from an interpolation between the nine HST fluxes and by using the calibration constants from de Ruyter \& Lub (1986). Extrapolation was necessary for the $L$ and $W$.

It turns out that the magnitude differences are equal for both photometric systems, and amount to 2 . 7 for the visual, 3 . 2 for

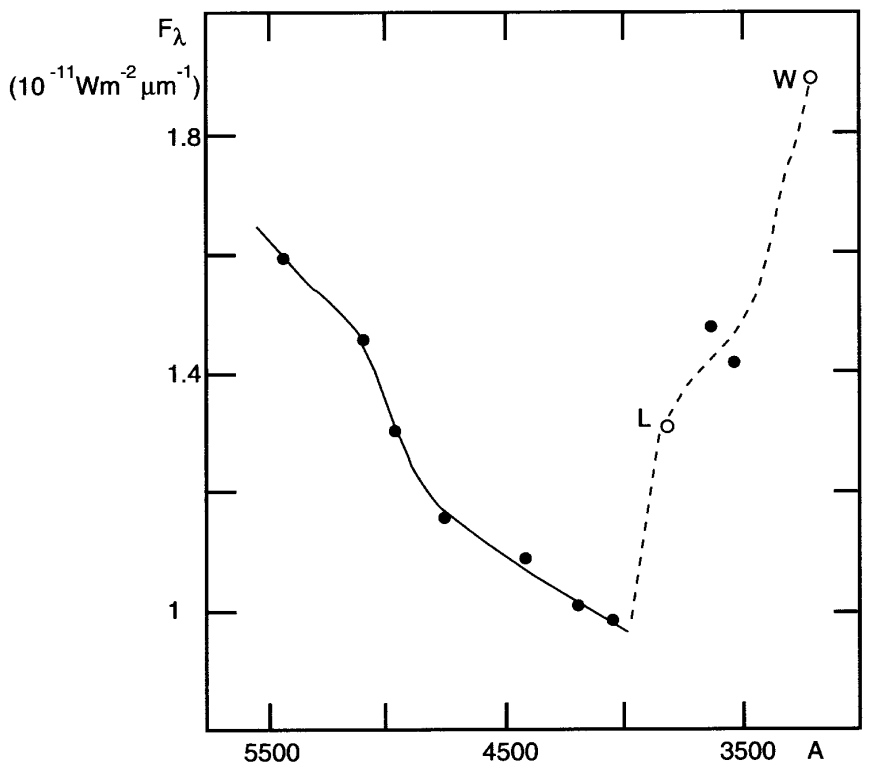

Fig. 4. The HST fluxes of Component A taken from Davidson et al. (1995) (dots) and two computed fluxes for the near-UV passbands $L$ and $W$ of the $V B L U W$ system.

the blue and 2.9 for the ultraviolet. Thus, the Homunculus is about 15 times brighter than the apparent light directly received from Component $\mathrm{A}$.

It is now easy to calculate the continuum fluxes of Component $\mathrm{A}$ at the effective wavelenghts of $L$ and $W$ (3837 $\AA$ and $3235 \AA$ ), respectively, as if they had been measured by the HST. The results are for $L$ and $W: 1.31 \times$ $10^{-11} \mathrm{~W} \mathrm{~m}^{-2} \mu \mathrm{m}^{-1}$ and $1.89 \times 10^{-11} \mathrm{~W} \mathrm{~m}^{-2} \mu \mathrm{m}^{-1}$, respectively. These data points are plotted as circles in Fig. 4 with the HST fluxes as dots. The steep rising trend for the near-UV, suggesting a maximum beyond the $W$ passband, points to a strong Balmer continuum radiation field.

The general impression is that Component $\mathrm{A}$ has a peak in the UV. A high flux in the far-UV has been also found by the Far Ultraviolet Spectroscopic Explorer satellite (Iping et al. 2001). These authors conclude that the observed far-UV spectrum $(920-1190 \AA)$ is probably formed in an extended $\left(1^{\prime \prime}-\right.$ 2 " diameter) UV scattering envelope.

That the (bi-polar) stellar wind should be responsible for the bulk of that radiation in emission lines and Balmer continuum is evident from spectroscopic studies (e.g. Hillier et al. 2001). This wind probably consists amongst others of an extended equatorial disk (e.g. Damineli et al. 1998; Ishibashi et al. 1999; van Genderen et al. 1999; Gull \& Ishibashi 2001; Duncan \& White 2003).

\section{Conclusions}

Evidence is accumulating that influential stellar colliding winds, mass flow variations as well as oscillations of the S Dor primary are likely responsible for many photometric features (van Genderen et al. 1994a,b, 1995, 1999, 2001). The flares discussed in the present paper are a special example, and they are terminated by a sharp dip pointing to an eclipse or obscuration phenomenon. Like the photometric studies in the optical, 
those in the NIR (Feast et al. 2001) as well as the many spectroscopic, X-ray and radio studies suggest the presence of a complicated gaseous environment around the central star(s).

We derived the magnitude differences between Component $\mathrm{A}$ and the flares as $\sim 2 \mathrm{~m} .5$ in the optical. Since we have no detailed information on the spectrum nor on the foreground reddening in the Homunculus, no luminosity could be derived. In case of a mass-losing binary an estimate based on the kinetic energy transformation formula points to $\sim 10^{5} L_{\odot}$, which is of the same order as derived from the brightness of the flares.

The HST fluxes of Component A, supplemented with the calculated continuum fluxes in the $L(3837 \AA)$ and $W(3235 \AA)$ bands of the $V B L U W$ system, show a strong UV excess due to Balmer continuum radiation by the stellar wind.

Acknowledgements. A.M.v.G. thanks V. Icke for useful discussions. C.S. acknowledges a research grant from the Belgian Fund for Scientific Research. This work has been supported by "IUAP P5/36" Interuniversity Attraction Poles Programme of the Belgian Federal Office for Scientific, Technical and Cultural Affairs, and the Belgian Fund for Scientific Research (FWO).

\section{References}

Allen, W. H. 1989, Southern Stars, 32, 159

Corcoran, M. F., Fredericks, A. C., Petre, R., et al. 2000, ApJ, 545, 420

Corcoran, M. F., Ishibashi, K., Swank, J. H., \& Petre, R. 2001a, ApJ, 547,1034

Corcoran, M. F., Ishibashi, K., Swank, J. H., \& Petre, R. 2001b, in Eta Carinae and other mysterious stars: the hidden opportunities of emission line spectroscopy, ed. T. R. Gull, S. Johansson, \& K. Davidson, ASP Conf. Ser., 242, 41

Cousins, A. W. J., \& Warren, P. R. 1963, MNASSA, 22, 65

Damineli, A. 1996, ApJ, 460, L49

Damineli, A., Kaufer, A., Wolf, B., et al. 2000, ApJ, 528, L101

Damineli, A., Stahl, O., Kaufer, A., et al. 1998, A\&AS, 132, 1

Davidson, K. 1999, in Eta Carinae at the millennium, ed. J. A. Morse, R. M. Humphreys, \& A. Damineli, ASP Conf. Ser., 179, 304

Davidson, K., \& Humphreys, R. M. 1997, ARA\&A, 35, 1

Davidson, K., Gull, T., Humphreys, R. M., et al. 1999, AJ, 118, 1777

Davidson, K., Ebbets, D., Weigelt, G., et al. 1995, AJ, 109, 1784

Davidson, K., Ishibashi, K., Gull, T., et al. 2000, ApJ, 530, L107

Duncan, R. A., White, S. M., Reynolds, J. E., \& Lim, J. 1999, in Eta Carinae at the millennium, ed. J. A. Morse, R. M. Humphreys, \& A. Damineli, ASP Conf. Ser., 179, 54

Duncan, R. A., \& White, S. M. 2003, MNRAS, 338, 425

Feast, M. W., Whitelock, P., \& Marang, F. 2001, MNRAS, 322, 741

Feinstein, A., \& Marraco, H. G. 1974, A\&A, 30, 271

van Genderen, A. M. 2001, A\&A, 366, 508

van Genderen, A. M., de Groot, M., \& Thé, P. S. 1994a, A\&A, 283, 89

van Genderen, A. M., de Groot, M., \& Thé, P. S. 1994b, in The impact of long-term monitoring on variable star research, ed. C. Sterken, \& M. de Groot, NATO ASI Ser. (Kluwer), 19

van Genderen, A. M., Sterken, C., de Groot, M., et al. 1995, A\&A, 304,415 van Genderen, A. M., Sterken, C., de Groot, M., \& Burki, G. 1999, A\&A, 343, 847

van Genderen, A. M., de Groot, M., \& Sterken, C. 2001, in P Cygni 2000: 400 years of progress, ed. M. de Groot, \& C. Sterken, ASP Conf. Ser., 233, 55

Gull, T. R., \& Ishibashi, K. 2001, in Eta Carinae and other mysterious stars: The hidden opportunities of emission line spectroscopy, ed. T. R. Gull, S. Johansson, \& K. Davidson, ASP Conf. Ser., 242, 59

Hillier, D. J., Davidson, K., Ishibashi, K., \& Gull, T. R. 2001, ApJ, 553, 837

Hoffleit, D. 1982, The Bright Star Catalogue, 4th ed. (New Haven: Yale Univ. Press)

Iping, R., Sonneborn, G., Gull, T. R., et al. 2001, in Eta Carinae and other mysterious stars: The hidden opportunities of emission line spectroscopy, ed. T. R. Gull, S. Johansson, \& K. Davidson, ASP Conf. Ser., 242, 55

Ishibashi, K., Corcoran, M. F., Davidson, K., et al. 1999, ApJ, 524, 983

Kholopov, P. N., Samus̀, N. N., Frolov, M. S., et al. 1985, General Cat. Var. Stars I (Moscow: Nauka)

Kukarkin, B. V., Kholopov, P. N., Artiukhina, N. M., et al. 1982, New Cat. of Suspected Var. Stars (Moscow: Nauka)

Lang, K. R. 1998, in Astrophys. Formulae II (Springer), 193

Manfroid, J., Sterken, C., Cunow, B., et al. 1994, A\&AS, 109, 329

Menzies, J. W., Cousins, A. W. J., Banfield, R. M., \& Laing, J. D. 1989, South African Astron. Obs. Circ., 32, 1

Mermilliod, J.-C., Mermilliod, M., \& Hauck, B. 1997, A\&AS, 124, 349

Pel, J. W. 1987, Internal Report, Leiden Obs.

Pittard, J. M., \& Corcoran, M. F. 2002, A\&A, 383, 636

de Ruyter, H. R., \& Lub, J. 1986, A\&AS, 63, 59

Slettebak, A. 1982, ApJS, 50, 55

Sterken, C. 2000, IBVS, 5000

Sterken, C., Freyhammer, L. M., Arentoft, T., \& van Genderen, A. M. 2001a, in P Cygni 2000: 400 years of progress, ed. M. de Groot, \& C. Sterken, ASP Conf. Ser., 233, 39

Sterken, C., Freyhammer, L. M., Arentoft, T., et al. 2001b, in P Cygni 2000: 400 years of progress, ed. M. de Groot, \& C. Sterken, ASP Conf. Ser., 233, 71

Sterken, C., Freyhammer, L. M., Arentoft, T., \& van Genderen, A. M. 1999b, A\&AS, 346, L33

Sterken, C., van Genderen, A. M., de Groot, M., \& Burki, G. 1999a, in Eta Carinae at the millennium, ed. J. A. Morse, R. M. Humphreys, \& A. Damineli, ASP Conf. Ser., 179, 295

Sterken, C., de Groot, M., \& van Genderen, A. M. 1996, A\&AS, 116, 9

Sterken, C., Manfroid, J., Beele, D., et al. 1995, A\&AS, 113, 31

Stevens, I. R., \& Pittard, J. M. 1999, in Eta Carinae at the millennium, ed. J. A. Morse, R. M. Humphreys, \& A. Damineli, ASP Conf. Ser., 179, 54

Viotti, R. F., Antonelli, L. A., \& Corcoran, M. F. 2002, A\&A, 385, 874

Whitelock, P. A., Feast, M. W., Koen, C., et al. 1994, MNRAS, 270, 364

Whitelock, P. A., \& Laney, D. 1999, in Eta Carinae at the millennium, ed. J. A. Morse, R. M. Humphreys, \& A. Damineli, ASP Conf. Ser., 179, 258

Wolf, B., Kaufer, A., Stahl, O., \& Damineli, A. 1999, in Eta Carinae at the millennium, ed. J. A. Morse, R. M. Humphreys, \& A. Damineli, ASP Conf. Ser., 179, 243

Zanella, R., Wolf, B., \& Stahl, O. 1984, A\&A, 137, 79 